\title{
AN ELLIPSOIDAL STATISTICAL MODEL FOR GAS MIXTURES*
}

\author{
STÉPHANE BRULL ${ }^{\dagger}$
}

\begin{abstract}
In this paper, we propose a construction of a new BGK model generalizing the Ellipsoidal Statistical Model [P. Andries, P. LeTallec, J.P. Perlat, B. Perthame, Eur. J. Mech. (B fluids), 19, 813-830, 2000], [L.H. Holway, Phys. Fluids, 9, 1958-1673, 1966] to the context of gas mixtures. The derivation of the model is based on the introduction of relaxation coefficients associated to some moments and the resolution of a minimization problem as in [S. Brull, J. Schneider, Cont. Mech. Thermodyn., 20(2), 63-74, 2008], [S. Brull, J. Schneider, Cont. Mech. Thermodyn., 20(8), 489-508,2009], [S. Brull, V. Pavan, J. Schneider, Eur. J. Mech. (B-Fluids), 33, 74-86, 2012]. We obtain in this work an ESBGK model for gas mixtures satisfying the fundamental properties of the Boltzmann collision operator (conservation laws, $\mathrm{H}$ theorem, equilibrium states, ...) and that is able to give a range of Prandtl numbers including the indifferentiability situation.
\end{abstract}

Key words. Kinetic theory, gas mixtures, BGK models, moments systems.

AMS subject classifications. 35Q20, 35Q35.

\section{Introduction}

The complexity of the nonlinear Boltzmann operator suggests to introduce simpler kinetic models. Hence, the BGK model [17] which is a well known simplified kinetic relaxation model has been introduced. Its features are to replace the complicated integral of collisions by a relaxation model while keeping some important physical and mathematical properties of the interaction term (conservation laws, $\mathrm{H}$ theorem, equilibrium states, ... ). The interest of these models is that they are easier to handle numerically and less costly at a computational point of view. However the extension of the BGK model to multi-component gases meets fundamental difficulties. For example, the hydrodynamic limit is much more complicated because phenomena such as diffusion or thermal diffusion must be considered.

Many BGK models have been proposed up to now, but they are still unsatisfactory and have been constructed on the same manner. That is they must reproduce the rates of exchanges of impulsion and energy of the Boltzmann operator for Maxwell molecules between the different species. For example one polynomial model has been proposed in [13]. But its relaxation function being a polynomial, the nonnegativity of the distribution function is not satisfied. However this model satisfies the indifferentiability principle stated in [13] inherited from the Boltzmann operator. That is when all the molecules have the same masses and their cross sections are equal, then the system of equations reduces to a single one by adding the distribution functions. A BGK model enjoying good mathematical properties has been derived in [1]. The main idea is to introduce only one BGK operator per species whose macrosopic parameters reproduce the interaction between each species with the others. But this model leads to incorrect transport coefficients at the hydrodynamic limit [21]. In [21], the authors constructed a BGK model which coincides with the Grad moments of the linearized Boltzmann operator. However the relaxation function of the model being polynomial, the nonnegativity of the distribution function is not ensured.

Hence the aim of this paper is to construct a BGK model that is able to recover

\footnotetext{
*Received: July 16, 2013; accepted (in revised form): October 9, 2013. Communicated by Benoit Perthame.

†Univ. Bordeaux, IMB, UMR 5251, F-33400 Talence, France (Stephane.Brull@math.ubordeaux1.fr).
} 
correct transport coefficients and enjoys good mathematical properties. The question is important because in general the authors compute the hydrodynamic limit from their own model and eventually compare with the correct fluid model [18]. In the situation of single and monospecies a BGK model leading to the correct Prandtl number has been proposed in [19]. This model is called Ellipsoidal Statistical Model (ESBGK). But the proof of the $\mathrm{H}$ theorem has been shown later on [2]. Recently, a new approach of such a model has been proposed in [7] based on the an entropy minimization principle $[22,23,20]$. The philosophy of the derivation consists in defining different relaxation rates associated to some moments of the distribution function. These rates intuitively describe the way in which these moments vanish for the trend to equilibrium. For example, defining a relaxation rate on the viscous stress tensor allows one to reproduce viscosity and heat flux. Generally, the moment that is dissipated is considered with respect to the polynomial function that is involved in the computation of the corresponding transport coefficient that we aim to fit. In [8], this approach has been generalized to the polyatomic context to propose a new construction of the Ellipsoidal Statistical Model [2]. In that case, another constraint of equalization of translational and internal temperature allows to simultaneously fit shear viscosity, volume viscosity, and heat flux. In the context of gas mixtures, things are more complicated. Indeed, there are more transport coefficients, that is the Fick, Soret, Dufour, Fourier, and Newton coefficients (see (4.6)). In [10], this method has been considered for gas mixtures by deriving a BGK model that is able to recover the correct Fick law. In this situation, several relaxation parameters are involved and are shown to be directly related to the non zero eigenvalues of the diffusion part of the Onsager matrix. Moreover even if Soret, Dufour, and Fourier coefficients are not correct, the structure of the fluxes is recovered. In [9], this model has been generalized to the slow chemistry framework where the mechanical process dominates the chemical one. The BGK model is then defined as a splitting between the mechanical model of [10] and the chemical one constructed in [14]. Next a Navier-Stokes system is constructed for the weak reaction regime from a Chapman-Enskog procedure and the Fick matrix is shown to be recovered by the model.

The present paper is organized as follows. In Section 2, we clarify some notations and recall some backgrounds about the Boltzmann operator for gas mixtures. The third section is devoted to the construction and the fundamental properties of the ESBGK model for gas mixtures. Firstly, we introduce the set of constraints. Next, we solve a minimization problem under moments constraints and we prove that the resulting model satisfies an $\mathrm{H}$ theorem and the indifferentiability principle. In Section 4, we perform a Chapman-Enskog expansion up to Navier-Stokes level. At this step, the two relaxation coefficients are computed in order to get an exact viscosity and an exact heat flux. Finally Section 5 gives a conclusion and perspectives on this work.

\section{Notations and some background about kinetic equations for gas mix-} tures

2.1. Boltzmann equation for gas mixtures. In this paper, we consider a gas mixtures with $p$ components. This mixture can be described at the kinetic level by its distribution function $\mathbf{f}=\left(f_{1}, \ldots, f_{p}\right)$, where $f_{i}(t, x, v)$ represents the distribution function of the species $i$. Here, $t$ corresponds to the time variable, $x$ to the space variable, and $v$ to the velocity variable. The distribution function for the mixture evolves according to the Boltzmann equation for a $p$ component gas 


$$
\forall i \in[1, p], \partial_{t} f_{i}+\mathbf{v} \cdot \nabla_{\mathbf{x}} f_{i}=\sum_{k=1}^{k=p} Q_{k i}\left(f_{k}, f_{i}\right):=Q_{i}(\mathbf{f}, \mathbf{f})
$$

where

$$
Q_{k i}\left(f_{k}, f_{i}\right)=\int_{\mathbb{R}^{3} \times \mathbb{S}^{2}}\left(f_{k}\left(\mathbf{w}_{k i}^{*}\right) f_{i}\left(\mathbf{v}_{k i}^{*}\right)-f_{k}(\mathbf{w}) f_{i}(\mathbf{v})\right) \sigma_{i k}(\boldsymbol{\omega} \cdot \mathbf{V},\|\mathbf{V}\|)\|\mathbf{V}\| d \mathbf{w} d \boldsymbol{\omega} .
$$

Here $Q_{k i}$ is the Boltzmann collision operator between molecules of species $i$ and $k$ and $\sigma_{i k}=\sigma_{k i}$ is the differential cross section which depends on the interaction potential between species $i$ and $k$. Finally $\mathbf{V}=\mathbf{w}-\mathbf{v}$ is the relative velocity. The post collisional velocities are given by

$$
\mathbf{v}_{k i}^{*}=\mathbf{v}-2 \frac{m_{k}}{m_{i}+m_{k}}((\mathbf{v}-\mathbf{w}) \cdot \boldsymbol{\omega}) \boldsymbol{\omega}, \quad \mathbf{w}_{k i}^{*}=\mathbf{w}+2 \frac{m_{i}}{m_{i}+m_{k}}((\mathbf{v}-\mathbf{w}) \cdot \boldsymbol{\omega}) \boldsymbol{\omega} .
$$

Those equations satisfy the conservation of momentum and energy at a microscopic level,

$$
m_{i} \mathbf{v}+m_{k} \mathbf{w}=m_{i} \mathbf{v}_{k i}^{*}+m_{k} \mathbf{w}_{k i}^{*}, \quad m_{i}\|\mathbf{v}\|^{2}+m_{k}\|\mathbf{w}\|^{2}=m_{i}\left\|\mathbf{v}_{k i}^{*}\right\|^{2}+m_{k}\left\|\mathbf{v}_{k i}^{*}\right\|^{2} .
$$

2.2. Macroscopic quantities. We denote by $n^{i}, \rho^{i}, u^{i}, E^{i}, T^{i}$, and $E^{i}$ the macroscopic quantities representing respectively the number density, density, average velocity, energy per unit volume, energy per particle, and finally temperature of a given species $i$. They are defined by the relations

$$
\begin{array}{r}
n^{i}=\int_{\mathbb{R}^{3}} f_{i} d v, \rho^{i}=m_{i} n^{i}, n^{i} \mathbf{u}^{i}=\int_{\mathbb{R}^{3}} v f_{i} d v, E^{i}=\frac{1}{2} \rho^{i}\left(\mathbf{u}^{i}\right)^{2}+n^{i} \mathcal{E}^{i}, \\
\mathcal{E}^{i}=\frac{3}{2} k_{B} T^{i}=\frac{m_{i}}{2 n^{i}} \int_{\mathbb{R}^{3}}\left(v-\mathbf{u}^{i}\right)^{2} f_{i} d v,
\end{array}
$$

where $m_{i}$ represents the molecular mass of the species $i$ and $k_{B}$ is the Boltzmann constant. In the same way, we define the hydrodynamic quantities for the mixture

$$
n=\sum_{k=1}^{p} n^{k}, \quad \rho=\sum_{k=1}^{p} \rho^{k}, \quad \rho \mathbf{u}=\sum_{k=1}^{p} \rho^{k} \mathbf{u}^{k}, \quad n \mathcal{E}+\frac{\rho}{2} \mathbf{u}^{2}=E=\sum_{k=1}^{p} E^{k}, \quad \mathcal{E}=\frac{3}{2} k_{B} T .
$$

$n^{i}, \mathbf{u}$, and $T$ being given, we define the Maxwellian distribution functions as

$$
\forall i \in[1, p], \quad \mathcal{M}_{i}=\frac{n^{i}}{\left(2 \pi k_{B} T / m_{i}\right)^{\frac{3}{2}}} \exp \left(-\frac{m_{i}(v-\mathbf{u})^{2}}{2 k_{B} T}\right) .
$$

The entropy associated to the distribution function $\mathbf{f}$ of the mixture is defined by

$$
\mathcal{H}(\mathbf{f})=\sum_{i=1}^{p} \int_{\mathbb{R}^{3}}\left(f_{i} \ln \left(f_{i}\right)-f_{i}\right) d v
$$


2.3. Other considerations. Using the above notations we denote by $\mathbb{L}^{2}(\mathcal{M})$ the set of measurable functions $\boldsymbol{\Psi}=\left(\psi_{1}, \cdots, \psi_{p}\right)$ such that

$$
\|\mathbf{\Psi}\|^{2}:=\sum_{i=1}^{i=p} \int_{\mathbb{R}^{3}} \psi_{i}^{2} \mathcal{M}_{i}<+\infty .
$$

This space is equipped with its natural dot product:

$$
\langle\boldsymbol{\Psi}, \mathbf{\Phi}\rangle=\sum_{i=1}^{i=p} \int_{\mathbb{R}^{3}} \psi_{i} \phi_{i} \mathcal{M}_{i} d v .
$$

In the sequel we often use the dot product notation. This notation is valid both for lists of $p$ scalar functions and for $p$ tensorial functions. For instance if $\boldsymbol{\Psi}, \boldsymbol{\Phi}$ are two lists of $p$ (symmetrical) tensorial functions $\psi_{i}, \phi_{i}, i \in[1, p]$, then the dot product notation $\langle\psi, \phi\rangle$ should be understood as

$$
\langle\psi, \phi\rangle:=\sum_{i=1}^{i=p} \int_{\mathbb{R}^{3}} \psi_{i} \otimes \phi_{i} \mathcal{M}_{i} d \mathbf{v},
$$

where $\otimes$ denotes the usual tensorial product. Note also the following convention: assume that $\mathbf{V}$ is a list of $p$ vectors and $\mathbf{T}$ a list of $p$ square matrices. Then a notation like $\alpha \mathbf{V}, \alpha \mathbf{T}$ (where $\alpha$ is a scalar) means that the scalar $\alpha$ is distributed on each line of the list of vectors or matrix. Besides if $\beta$ is a vector, then a notation like $\beta \cdot \mathbf{V}$ means that we distribute the dot product by $\beta$ on each line of the vector $\mathbf{V}$. In the same way, if $\gamma$ is a square matrix, then a notation like $\boldsymbol{\gamma}: \mathbf{T}$ means that we distribute the (total) dot product (between matrix) by $\boldsymbol{\gamma}$ on each line of the line of $\mathbf{T}$. Finally, if $\mathbf{s}$ is a tensor and $\mathbf{S}$ a list of $p$ tensors, then a notation like $\mathbf{s} \otimes \mathbf{S}$ means that we distribute the tensor product by $\mathbf{s}$ on the left on each line of the list $\mathbf{S}$.

Definition 2.1. The set $\mathbb{K}$ belonging to $\mathbb{L}^{2}(\mathcal{M})$ of the collision invariants of the Boltzmann operator (2.1) is spanned by the following list of functions:

$$
\left(\begin{array}{c}
1 \\
0 \\
\vdots \\
0
\end{array}\right), \cdots,\left(\begin{array}{c}
0 \\
0 \\
\vdots \\
1
\end{array}\right),\left(\begin{array}{c}
m_{1} v_{x} \\
m_{2} v_{x} \\
\vdots \\
m_{p} v_{x}
\end{array}\right),\left(\begin{array}{c}
m_{1} v_{y} \\
m_{2} v_{y} \\
\vdots \\
m_{p} v_{y}
\end{array}\right),\left(\begin{array}{c}
m_{1} v_{z} \\
m_{2} v_{z} \\
\vdots \\
m_{p} v_{z}
\end{array}\right),\left(\begin{array}{c}
m_{1} \boldsymbol{v}^{2} \\
m_{2} \boldsymbol{v}^{2} \\
\vdots \\
m_{p} \boldsymbol{v}^{2}
\end{array}\right) .
$$

This space is of dimension $p+4$. We denote by $\boldsymbol{\phi}^{l}, l \in[1, p+4]$ the list of these $p+4$ elements.

In contrast to the case of monatomic gas there exists a space $\mathbb{C}$ of moments of degree 1 in velocity which is not conserved. This space is defined as follows.

Definition 2.2. Let $\boldsymbol{C}_{i}$ be the vector whose $i^{\text {th }}$ component is $\boldsymbol{v}-\boldsymbol{u}$ and others are 0 . Denote by $\mathcal{P}_{\mathbb{K}}$ the orthogonal projection on $\mathbb{K}$ and let $\mathcal{I}$ be the identity operator. Then we define $\mathbb{C}$ as the space generated by the vectors $\left(\mathcal{I}-\mathcal{P}_{\mathbb{K}}\right)\left(\boldsymbol{C}_{i}\right), i \in[1, p]$.

In [10], we have shown the following lemma which exhibits a basis of $\mathbb{C}$.

Lemma 2.3. The family $\left(\mathcal{I}-\mathcal{P}_{\mathbb{K}}\right)\left(\boldsymbol{C}_{i}\right), i \in[1, p]$ is composed of $p-1$ independent "vectors" and as a consequence the dimension of $\mathbb{C}$ is $3(p-1)$. 
For the Chapman-Enskog procedure, introduce the Sonine polynomials $\mathbb{A}$ and $\mathbf{B}$ whose $i^{\text {th }}$ components are

$$
\mathbb{A}_{i}=m_{i}\left(v \otimes v-\frac{1}{3} v^{2} I d\right), \quad B_{i}=(v-\mathbf{u})\left(\frac{1}{2} m_{i}(v-\mathbf{u})^{2}-\frac{5}{2} k_{B} T\right) .
$$

\section{Construction and properties of the model}

3.1. Fundamental properties of kinetic operators. As in $[1,10]$, the BGK model $\mathcal{R}(\mathbf{f})$ constructed in this paper consists in one operator per species as

$$
\mathcal{R}(\mathbf{f})=\left(\mathcal{R}_{1}(\mathbf{f}), \ldots, \mathcal{R}_{p}(\mathbf{f})\right) .
$$

We present now the fundamental properties coming from the Boltzmann operator for gas mixtures that our BGK model has to satisfy.

\section{Collisional invariants}

$$
\forall \mathbf{f}, f_{i} \geq 0, \forall \boldsymbol{\phi}, \sum_{i=1}^{i=p} \int_{\mathbb{R}^{3}} \mathcal{R}_{i}(\mathbf{f}) \phi_{i} d \mathbf{v}=0 \Leftrightarrow \boldsymbol{\phi} \in \mathbb{K} .
$$

2. H-theorem: for any list of nonnegative functions $\mathbf{f}=\left(f_{1}, \ldots, f_{p}\right)$,

$$
\sum_{i=1}^{i=p} \int_{\mathbb{R}^{3}} \mathcal{R}_{i}(\mathbf{f}) \ln \left(f_{i}\right) d \mathbf{v} \leq 0
$$

3. Equilibrium states: the equality holds in the above equation if and only if $\mathbf{f}$ is at thermodynamic equilibrium, i.e. there exists macroscopic values $n_{1}, \ldots, n_{p}, \mathbf{u}$, and $\mathrm{T}$ such that

$$
\forall i \in[1, p], f_{i}=\mathcal{M}_{i} .
$$

In such a case we denote $\mathbf{f}=\mathcal{M}$. Moreover $\mathcal{M}$ is the only set of functions such that

$$
\mathcal{R}_{i}(\mathbf{f})=0 .
$$

3.2. Space of constraints. $\mathbf{f}$ being given, $\mathbf{G}$ is researched as the solution of an entropy minimisation problem under moments constraints. The space of constraints that is used in this paper is given in the following definition.

Definition 3.1. The space of constraints $\mathbf{K}(\mathbf{f})$ associated to $\mathbf{f}$ is defined by the set of functions $\mathbf{g}=\left(g_{1}, \ldots, g_{p}\right)$ s.t. $\mathbf{g} \geq 0$ a.e. and satisfies

$$
\forall l \in\{1, p+4\}, \sum_{i=1}^{p} \int_{\mathbb{R}^{3}} \phi_{i}^{l}\left(g_{i}-f_{i}\right) d v=0
$$

to obtain the conservation properties and the relaxation constraint

$$
\sum_{i=1}^{p} \int_{\mathbb{R}^{3}} \lambda\left(g_{i}-f_{i}\right) \mathbb{A}_{i}(v-\mathbf{u}) d v=-\lambda_{1} \sum_{i=1}^{p} \int_{\mathbb{R}^{3}} f_{i} \mathbb{A}_{i}(v-\mathbf{u}) d v
$$

where $\lambda>0$ and $\lambda_{1}>0$ are free parameters that are devoted to fit the transport coefficients. Like in [7, 8, 10], they are fixed during the Chapman-Enskog procedure. 
The constraint (3.6) can be rewritten as

$$
\frac{1}{\rho} \sum_{i=1}^{p} \int_{\mathbb{R}^{3}} m_{i} g_{i}(v-\mathbf{u}) \otimes(v-\mathbf{u}) d v=\left(1-\frac{\lambda_{1}}{\lambda}\right) \Theta+\frac{\lambda_{1}}{\lambda} T
$$

where $\Theta$ represents the pressure tensor defined by

$$
\Theta=\sum_{i=1}^{p} \int_{\mathbb{R}^{3}} m_{i}(v-\mathbf{u}) \otimes(v-\mathbf{u}) f_{i} d v
$$

In order to compare our model with the one derived in $[2,7]$, we use the change of variable $\nu=1-\frac{\lambda_{1}}{\lambda}$. So the constraint (3.7) on $\mathbf{g}$ can be rewritten

$$
\frac{1}{\rho} \sum_{i=1}^{p} \int_{\mathbb{R}^{3}} m_{i} g_{i}(v-\mathbf{u}) \otimes(v-\mathbf{u}) d v=\nu \Theta+(1-\nu) T=\tau .
$$

REMARK 3.1. In [10], the relaxation constraints (3.6) are written on a basis of $\mathbb{C}$ instead of the Sonine polynomials (2.4). This basis is obtained from the diagonalization of the diffusion part of the Onsager matrix.

THEOREM 3.2. For any nonnegative function $\mathbf{f}=\left(f_{1}, \ldots, f_{p}\right)$ and $\nu \in\left[-\frac{1}{2}, 1[\right.$, the tensor $\tau$ defined by 3.9 is symmetric positive definite and the minimization entropy problem

$$
\mathbf{G}=\min _{\mathbf{g} \in \mathbf{K}(\mathbf{f})} \mathcal{H}(\mathbf{g})
$$

has a unique solution $\mathbf{G}=\left(G_{1}, \ldots, G_{p}\right)$, where $G_{i}$ writes

$$
G_{i}=\frac{\rho_{i}}{\sqrt{2 \pi \operatorname{det}(\tau)}} \exp \left(-\frac{1}{2}\left\langle v-\mathbf{u}, \tau^{-1}(v-\mathbf{u})\right\rangle\right) .
$$

Conversely, if the minimization problem (3.10) has a unique solution for any nonnegative function $\mathbf{f} \in L_{1}^{2}$, then $\nu \in\left[-\frac{1}{2}, 1[\right.$.

Proof. To prove the existence of the distribution function (3.11), we have to solve a minimization problem under moments constraints. As in [10], we follow the methodology of Junk [20]. Hence we consider the set

$$
\begin{aligned}
\Lambda= & \left\{\left(\alpha_{l}\right)_{l},\left(\lambda_{r}\right)_{r} /\right. \\
& \left.\int \exp \left(\sum_{l=1}^{p+3} \alpha_{l} \phi_{i}^{l}+\lambda_{1} v_{1}^{2}+\lambda_{2} v_{2}^{2}+\lambda_{3} v_{3}^{2}+\lambda_{4} v_{1} v_{2}+\lambda_{5} v_{1} v_{2}+\lambda_{6} v_{2} v_{3}\right)<+\infty\right\} .
\end{aligned}
$$

If this set is open, then the minimization problem has a unique solution as soon as $\mathbf{K}(\mathbf{f}) \neq \emptyset$. This last condition is fulfilled because $\mathbf{G}$ defined by (3.11) belongs to $\mathbf{K}(\mathbf{f})$. Hence it remains to show that (3.12) is open. Proving this assertion is equivalent to showing that the signature of the quadratic form

$$
q(v)=\lambda_{1} v_{1}^{2}+\lambda_{2} v_{2}^{2}+\lambda_{3} v_{3}^{2}+\lambda_{4} v_{1} v_{2}+\lambda_{5} v_{1} v_{2}+\lambda_{6} v_{2} v_{3}
$$


is $(0,3)$. Introducing the matrix associated to the quadratic form $q, M=\left(m_{i j}\right)_{i j}$, the Gauss reduction of $q(3.13)$ writes

$$
\begin{array}{r}
q(v)=m_{11}\left(v_{1}+\frac{m_{12}}{m_{11}} v_{2}+\frac{m_{13}}{m_{11}} v_{3}\right)^{2}+\alpha\left(v_{2}+\frac{1}{\alpha}\left(m_{23}-\frac{m_{13}}{m_{11}}\right) v_{3}\right)^{2}+\gamma v_{3}^{2}, \\
m_{11}<0, \alpha<0, \gamma<0,
\end{array}
$$

where

$$
\alpha=m_{22}-\frac{m_{12}^{2}}{m_{11}}, \gamma=m_{33}-\frac{m_{13}^{2}}{m_{11}}-\frac{1}{\alpha}\left(m_{23}-\frac{m_{12} m_{13}}{m_{11}}\right)^{2} .
$$

Hence for $\varepsilon>0$ small enough, we can show that the signatures of the quadratic form associated to the matrix whose terms are $m_{i j}+\varepsilon$ and $m_{i j}-\varepsilon$ is $(0,3)$. This means that the set (3.12) is open.

The proof of the second part of the theorem is analogous to the one given in the monospecies setting, so we refer to [7] for more details.

3.3. H Theorem. In this section, we show that the present BGK model is entropic for $-\frac{1}{2} \leq \nu<1$.

TheOREM 3.3. For $-\frac{1}{2} \leq \nu<1$ and $G_{i}$ defined by (3.11), we have the following entropy dissipation property:

$$
D(\mathbf{f})=\sum_{i=1}^{p}\left(G_{i}-f_{i}\right) \ln \left(f_{i}\right) d v \leq 0 .
$$

Moreover, $D(\mathbf{f}) \leq 0$ for $-\frac{1}{2} \leq \nu<1$ with equality if and only if $f_{i}=\mathcal{M}_{i}, i=$ $\{1, \ldots, p\}$.

Proof. The proof is similar to the one given in [7] but adapted to a gas mixture framework. For the sake of clarity, we give the proof in detail. By using the convexity of $x \mapsto x \ln (x)-x$, we get that

$$
\sum_{i=1}^{p} \int_{\mathbb{R}^{3}}\left(G_{i, \nu}-f_{i}\right) \ln f_{i} d v \leq \mathcal{H}\left(\mathbf{G}_{\nu}\right)-\mathcal{H}(\mathbf{f})
$$

where $G_{i, \nu}$ is the function defined in (3.11) and $\nu$ represents its dependance with respect to $\nu \in\left[-\frac{1}{2}, 1\left[\right.\right.$. Moreover, because $\mathbf{G}_{1-\varepsilon}$ is the result of a minimization problem under constraints of $f, \mathcal{H}\left(\mathbf{G}_{\mathbf{1}-\varepsilon}\right) \leq \mathcal{H}(\mathbf{f})$. So

$$
\sum_{i=1}^{p} \int_{\mathbb{R}^{3}}\left(G_{i, \nu}-f_{i}\right) \ln f_{i} d v \leq \mathcal{H}\left(\mathbf{G}_{\nu}\right)-\mathcal{H}\left(\mathbf{G}_{\mathbf{1}-\varepsilon}\right) .
$$

But a direct computation of $\mathcal{H}\left(\mathbf{G}_{\nu}\right)$ gives

$$
\mathcal{H}\left(\mathbf{G}_{\nu}\right)=\sum_{i=1}^{p} \rho_{i} \ln \left(\frac{\rho_{i}}{\sqrt{2 \pi \operatorname{det} \mathcal{T}}}\right)-\frac{5}{2} \rho .
$$

Moreover the set of constraints that is used for the definition of $\mathbf{G}_{\nu}$ are linear, and the application $\nu \mapsto G_{i, \nu}$ is convex. So to obtain the entropy dissipation property, it is enough to show that $\mathcal{H}\left(\mathbf{G}_{\nu}\right) \leq \mathcal{H}\left(\mathbf{G}_{\mathbf{1 - \varepsilon}}\right)$.

$$
\mathcal{H}\left(\mathbf{G}_{\nu}\right)-\mathcal{H}\left(\mathbf{G}_{\mathbf{1}-\varepsilon}\right)=\frac{1}{2} \rho(\ln (\operatorname{det}((1-\varepsilon) \Theta+\varepsilon T I d))-\ln (\operatorname{det}(\nu \Theta+(1-\nu) T I d))) .
$$


But this term has the same sign as

$$
p(\nu)=\operatorname{det}((1-\varepsilon) \Theta+\varepsilon T I d)-\operatorname{det}(\nu \Theta+(1-\nu) T I d) .
$$

$p(\nu)$ can be rewritten as

$$
p(\nu)=\left(\prod_{i=1}^{3}\left(\theta_{i}+\varepsilon\left(T-\theta_{i}\right)\right)+\prod_{i=1}^{3}\left(\nu\left(T-\theta_{i}\right)-T\right)\right) .
$$

Hence by setting $\tilde{\theta}_{i}=\theta_{i} / T$, we get

$$
p(\nu)=T^{3}\left(\prod_{i=1}^{3}\left(\tilde{\theta}_{i}+\varepsilon\left(1-\tilde{\theta}_{i}\right)\right)+\prod_{i=1}^{3}\left(\nu\left(1-\tilde{\theta}_{i}\right)-1\right)\right) .
$$

Then

$$
p\left(-\frac{1}{2}\right)=\left(\prod_{i=1}^{3}\left(\tilde{\theta}_{i}+\varepsilon\left(1-\tilde{\theta}_{i}\right)\right)+\prod_{i=1}^{3}\left(-\frac{1}{2}\left(1-\tilde{\theta}_{i}\right)-1\right)\right) .
$$

Therefore after some computations

$$
p\left(-\frac{1}{2}\right)=\frac{3 T^{3}}{8}\left(3\left(\tilde{\theta_{1}} \tilde{\theta_{2}} \tilde{\theta_{3}}-\tilde{\theta_{1}} \tilde{\theta_{2}}-\tilde{\theta_{1}} \tilde{\theta_{3}}-\tilde{\theta_{2}} \tilde{\theta_{3}}\right)\right)+\varepsilon l\left(\tilde{\theta_{1}}, \tilde{\theta_{2}}, \tilde{\theta_{3}}\right)
$$

with

$$
\begin{aligned}
l\left(\tilde{\theta_{1}}, \tilde{\theta_{2}}, \tilde{\theta_{3}}\right)=\left(1-\tilde{\theta_{1}}\right) \tilde{\theta_{2}} \tilde{\theta_{3}}+\tilde{\theta_{1}}\left(1-\tilde{\theta_{2}}\right) \tilde{\theta_{3}}+\tilde{\theta_{1}} \tilde{\theta_{2}}\left(1-\tilde{\theta_{3}}\right) \\
+\varepsilon\left(\left(1-\tilde{\theta_{1}}\right)\left(1-\tilde{\theta_{2}}\right) \tilde{\theta_{3}}+\left(1-\tilde{\theta_{1}}\right) \tilde{\theta_{2}}\left(1-\tilde{\theta_{3}}\right)+\tilde{\theta_{1}}\left(1-\tilde{\theta_{2}}\right)\left(1-\tilde{\theta_{3}}\right)\right) \\
+\varepsilon^{2}\left(1-\tilde{\theta_{1}}\right)\left(1-\tilde{\theta_{2}}\right)\left(1-\tilde{\theta_{3}}\right) .
\end{aligned}
$$

By using Lagrange multipliers, we get that the maximum of $p\left(-\frac{1}{2}\right)$ is obtained for $\tilde{\theta}_{1}=\tilde{\theta}_{2}=\tilde{\theta}_{3}=1$ and yields $p\left(-\frac{1}{2}\right)=0$.

3.4. Indifferentiability condition. In this section, we prove that our model satisfies the indifferentiability property stated in [13] and recalled in introduction.

Proposition 3.4. If all the molecular masses are equal $\left(\forall i \in\{1 ; p\}, m_{i}=m\right)$, then the distribution function $\mathbf{f}=\sum_{i=1}^{p} f_{i}$ is a solution of the Ellipsoidal Statistical Model for monospecies [2, 7]. Hence the model satisfies the indifferentiability principle.

The proof is trivial and performed by adding term by term each component of kinetic equations.

\section{Chapman-Enskog expansion}

4.1. The Navier-Stokes system for gas mixtures. The Navier-Stokes system for a mixture of $p$ components reads

$$
\begin{array}{r}
\forall i \in[1, p], \partial_{t} n^{i}+\nabla \cdot\left(n^{i} \mathbf{u}+\mathbf{J}_{i}\right)=0, \\
\partial_{t}(\rho \mathbf{u})+\nabla \cdot\left(\mathbb{P}+\rho \mathbf{u} \otimes \mathbf{u}+\mathbb{J}_{\mathbf{u}}\right)=0, \\
\partial_{t} E+\nabla \cdot\left(E \mathbf{u}+\mathbb{P}[\mathbf{u}]+\mathbb{J}_{\mathbf{u}}[\mathbf{u}]+\mathbf{J}_{q}\right)=0,
\end{array}
$$


where $\mathbf{J}_{i}, \mathbb{J}_{\mathbf{u}}$, and $\mathbf{J}_{\mathbf{q}}$ are respectively the mass, momentum, and heat fluxes. These fluxes can be expressed either from thermodynamics of irreversible process (TIP) [12] or from the phenomenological point of view. Of course these two approaches are strictly equivalent according to the definition of the chemical potential (4.5). As it has been explained in [10], the Chapman-Enskog procedure leads naturally to fluxes defined from the TIP assumption whereas coefficients appearing in phenomenological approach are obtained from experiments.

We first describe the transport coefficients obtained from the TIP point of view. In this case, the mass, impulsion, and energy fluxes write

$$
\begin{aligned}
& \mathbf{J}_{i}=\sum_{j=1}^{j=p} L_{i j} \nabla\left(\frac{-\mu_{j}}{T}\right)+L_{i \mathbf{q}} \nabla\left(\frac{1}{T}\right), \\
& \mathbf{J}_{\mathbf{q}}=\sum_{j=1}^{j=p} L_{\mathbf{q} j} \nabla\left(\frac{-\mu_{j}}{T}\right)+L_{\mathbf{q q}} \nabla\left(\frac{1}{T}\right), \\
& \mathbb{J}_{\mathbf{u}}=L_{\mathbf{u u}} \mathbb{D}(\mathbf{u}),
\end{aligned}
$$

where

$$
\mathbb{D}(\mathbf{u})=\frac{1}{2}\left(\nabla \mathbf{u}+(\nabla \mathbf{u})^{T}\right)-\frac{1}{3}(\nabla \cdot \mathbf{u}) \mathbb{I}
$$

represents the Reynolds tensor and

$$
-\frac{\mu_{i}}{T}=k_{B}\left(\ln \left(n^{i}\right)-\frac{3}{2} \ln \left(\frac{2 \pi k_{B} T}{m_{i}}\right)\right)
$$

is the chemical potential.

By using the phenomenological approach, mass and impulsion fluxes write with respect to densities and temperature gradients as

$$
\mathbf{J}_{i}=\sum_{j=1}^{j=p} D_{i j} \nabla n_{j}+D_{i T} \nabla T, \quad \mathbf{J}_{\mathbf{q}}=\sum_{j=1}^{j=p} D_{\mathbf{q} j} \nabla n_{j}-D_{\mathbf{q q}} \nabla T,
$$

where $D_{i j}$ and $D_{i T}, D_{\mathbf{q} j}$ and $D_{\mathbf{q q}}$ respectively denote the Fick, Soret, Duffour, and Fourier coefficients.

(4.4) and (4.6) are shown to be equivalent by plugging the expression (4.5) of $-\frac{\mu_{i}}{T}$ into (4.4).

4.2. Computation of the relaxation coefficients $\lambda$ and $\lambda_{1}$. We determine now the coefficients $\lambda$ and $\lambda_{1}$ in such a way that the Chapman-Enskog expansion up to order one leads to the true viscosity and the true heat flux. That is, the ones that have been computed from the Boltzmann equation or that have been obtain experimentally.

Hence we consider the system of kinetic equations

$$
\partial_{t} f_{i}+v \cdot \nabla f_{i}=\frac{\lambda}{\varepsilon}\left(G_{i}-f_{i}\right), \quad i \in\{1, \ldots, p\},
$$

where $\varepsilon$ is proportional to the Knudsen number. For each component $i$, the distribution function $f_{i}$ is expanded according to $f_{i}=\mathcal{M}_{i}+\varepsilon f_{i}^{(1)}+\ldots$.

For more details on Chapman-Enskog expansion for gas mixtures, we refer to $[11,10]$. According to [10], the fluxes write

$$
\mathbf{J}_{i}=\left\langle\mathbf{f}^{(\mathbf{1})}, \mathbf{C}_{\mathbf{i}}\right\rangle, \quad \mathbf{J}_{\mathbf{q}}=\left\langle\mathbf{f}^{(\mathbf{1})}, \mathbf{B}\right\rangle, \quad \mathbb{J}_{\mathbf{u}}=\left\langle\mathbf{f}^{(\mathbf{1})}, \mathbb{A}\right\rangle .
$$


From (4.7), $\mathbf{G}$ which depends on $\mathbf{f}$ is expanded around the Maxwellian distribution $\mathcal{M}$ according to

$$
\mathbf{G}=\mathcal{M}+\varepsilon \mathbf{G}^{(1)}+\ldots
$$

Using that $G_{i}(\mathcal{M})=\mathcal{M}_{i}$, the expression of $G_{i}^{(1)}$ is given by

$$
G_{i}^{(1)}=D G_{i}(\mathcal{M}) \cdot f_{i}^{(1)}=\lim _{\tau \rightarrow 0} \frac{G_{i}\left(\mathcal{M}+\tau \mathbf{f}^{1}\right)-\mathcal{M}_{i}}{\tau} .
$$

So the order 0 in (4.7) leads to

$$
\partial_{t} \mathcal{M}+v \cdot \nabla \mathcal{M}=\lambda\left(\mathbf{G}^{(1)}-\mathbf{f}^{(1)}\right) .
$$

But, from [10], we get the relation

$$
\begin{gathered}
\left(\partial_{t}+v \cdot \nabla\right) \mathcal{M}=\left(\sum_{j=1}^{p} k_{B}^{-1}\left(\mathcal{I}-\mathcal{P}_{\mathbb{K}}\right)\left(C_{j}\right) \cdot \nabla\left(-\frac{\mu_{j}}{k_{B} T}\right)+\frac{1}{k_{B} T} \mathbb{A}: \mathbb{D}(\mathbf{u})\right. \\
\left.+\frac{1}{k_{B}} \mathbf{B} \cdot \frac{\nabla T}{T^{2}}\right) \mathcal{M}
\end{gathered}
$$

where the Sonine polynomial $\mathbf{B}$ has been defined in (2.4). Therefore $\mathbf{f}^{(1)}$ has the following expression:

$$
\mathbf{f}^{(1)}=\mathbf{G}^{(1)}-\left(\sum_{j=1}^{p} k_{B}^{-1}\left(\mathcal{I}-\mathcal{P}_{\mathbb{K}}\right)\left(C_{j}\right) \cdot \nabla\left(-\frac{\mu_{j}}{k_{B} T}\right)+\frac{1}{k_{B} T} \mathbb{A}: \mathbb{D}(\mathbf{u})+\frac{1}{k_{B}} \mathbf{B} \cdot \frac{\nabla T}{T^{2}}\right) \mathcal{M}
$$

Relation (3.6) considered at order 1 for $\mathbf{g}=\mathbf{G}$ leads to

$$
\sum_{i=1}^{p} \int_{\mathbb{R}^{3}} \lambda\left(G_{i}^{(1)}-f_{i}^{(1)}\right) \mathbb{A}_{i}(v-\mathbf{u}) d v=-\lambda_{1} \sum_{i=1}^{p} \int_{\mathbb{R}^{3}} f_{i}^{(1)} \mathbb{A}_{i}(v-\mathbf{u}) d v .
$$

From orthogonality reasons,

$$
\begin{aligned}
\sum_{i=1}^{p} \int_{\mathbb{R}^{3}} f_{i}^{(1)} \mathbb{A}_{i}(v-\mathbf{u}) d v & =-\frac{1}{k_{B} T \lambda_{1}} \sum_{i=1}^{p} \int_{\mathbb{R}^{3}} \mathbb{A}_{i}(v-\mathbf{u}): \mathbb{A}_{i}(v-\mathbf{u}) \mathcal{M}_{i} d v \mathbb{D}(\mathbf{u}) \\
& =-\frac{n k_{B} T}{\lambda_{1}} \mathbb{D}(\mathbf{u}) .
\end{aligned}
$$

So we define $\lambda_{1}$ as $\lambda_{1}=\frac{n k_{B} T}{\mu}$. By setting

$$
\lambda=\frac{\lambda_{1}}{1-\nu}=\frac{\rho T}{\mu(1-\nu)},
$$

we get the relaxation operator (3.1):

$$
\mathcal{R}_{i}(\mathbf{f})=\frac{\rho T}{\mu(1-\nu)}\left(G_{i}-f_{i}\right), i \in\{1, \ldots, p\} .
$$


In order to determine the heat flux, we use (4.8). Multiply (4.10) by $\mathbf{B}(v-\mathbf{u})$ and integrate. From relation (4.9) defining $\mathbf{G}^{(1)}$, we can show that

$$
\int_{\mathbb{R}^{3}} \mathbf{G}^{(1)} \mathbf{B}(v-\mathbf{u}) d v=0 .
$$

By using the orthogonality of $\mathbf{B}$ with respect to $\mathbb{A}$ and $\left(\mathcal{I}-\mathcal{P}_{\mathbb{K}}\right)\left(C_{j}\right)$, it holds that

$$
\sum_{i=1}^{p} \int_{\mathbb{R}^{3}} B_{i}(v-\mathbf{u}) f_{i}^{(1)} d v=-\frac{1}{3 k_{B} \lambda} \sum_{i=1}^{p}\left(\int_{\mathbb{R}^{3}} B_{i}(v-\mathbf{u}) \cdot B_{i}(v-\mathbf{u}) \mathcal{M}_{i} d v\right) \frac{\nabla T}{T^{2}} .
$$

A direct computation gives

$$
\sum_{i=1}^{p} \int_{\mathbb{R}^{3}} B_{i}(v-\mathbf{u}) \cdot B_{i}(v-\mathbf{u}) \mathcal{M}_{i} d v=\frac{15}{2} k_{B}^{3} T^{3} \sum_{i=1}^{p} \frac{n^{i}}{m_{i}} .
$$

Hence, from (4.4) the heat flux $L_{\mathbf{q q}}$ has the following expression:

$$
L_{\mathbf{q q}}=\frac{5}{2 \lambda} k_{B}^{2} T^{3} \sum_{i=1}^{p} \frac{n^{i}}{m_{i}} .
$$

In order to compare the model constructed in the present paper, with the monospecies model derived in ([7]), we set $\kappa=\frac{L_{\mathbf{q q}}}{T^{2}}$. Hence, the Prandtl number writes

$$
\operatorname{Pr}=\frac{5}{2} \frac{R \mu}{\kappa}=R \frac{\lambda}{\lambda_{1}} \frac{n}{k_{B} \sum_{i=1}^{p} \frac{n^{i}}{m_{i}}} .
$$

Therefore by using the definition (4.13) of the relaxation coefficient $\lambda$ and $R=k_{B} / m$, we get

$$
\operatorname{Pr}=\frac{R}{1-\nu} \frac{n}{k_{B} \sum_{i=1}^{p} \frac{n^{i}}{m_{i}}}=\frac{1}{1-\nu} \frac{1}{\sum_{i=1}^{p} \frac{n^{i}}{n} \frac{m}{m_{i}}}
$$

where

$$
m=\sum_{i=1}^{p} \frac{n^{i}}{n} m_{i}
$$

represents the molecular mass of the mixture. Hence the present model is able to reach Prandtl numbers in the range

$$
\left[\frac{2}{3}\left(\sum_{i}^{p} \frac{n^{i}}{n} \frac{m}{m_{i}}\right)^{-1},+\infty[\right.
$$

Therefore for any Prandtl number in the interval (4.16), the relation (4.15) uniquely determines the coefficient $\lambda$ from the value of $\nu$.

For example, in the indifferentiability situation, the relation (4.15) becomes as in $[2,7], \operatorname{Pr}=1 /(1-\nu)$. Hence $\operatorname{Pr}=2 / 3$ is obtained for $\nu=-1 / 2$ and this totally determines $\lambda$. 
Next the other transport coefficients are computed. Their values are not correct, but the shape of the fluxes is recovered. In Property 4.1, we collect the values of all the transport coefficients.

PROPERTY 4.1. The transport coefficients are given by

$$
L_{i j}=\frac{3 T n^{i}}{k_{B} m_{i}} \delta_{i j}, \quad L_{i \mathbf{q}}=L_{\mathbf{q} i}=0, \quad L_{\mathbf{q q}}=\frac{5}{2 \lambda} k_{B}^{2} T^{3} \sum_{i=1}^{p} \frac{n^{i}}{m_{i}}, \quad L_{\mathbf{u u}}=-\mu .
$$

Proof. According to Chapmann-Enskog expansion, the mass flux writes $\mathbf{J}_{i}=$ $\left\langle\mathbf{f}^{(\mathbf{1})}, \mathbf{C}_{\mathbf{i}}\right\rangle$, where $\mathbf{f}^{(\mathbf{1})}$ is computed from (4.12). But, we have $\left\langle\mathbb{A}, \mathbf{C}_{i}\right\rangle=0,\left\langle\mathbf{B}, \mathbf{C}_{i}\right\rangle=0$. Then $L_{i q}=L_{q i}=0$ and

$$
L_{i j}=-\frac{1}{k_{B}} \int_{\mathbb{R}^{3}} \mathbf{C}_{i} \cdot \mathbf{C}_{j} \mathcal{M}_{i} d v=-\frac{1}{k_{B}} \int_{\mathbb{R}^{3}}\left(\mathcal{I}-\mathcal{P}_{\mathbb{K}}\right)\left(\mathbf{C}_{i}\right) \cdot\left(\mathcal{I}-\mathcal{P}_{\mathbb{K}}\right)\left(\mathbf{C}_{j}\right) \mathcal{M}_{i} d v .
$$

So the expression of $L_{i j}$ follows.

\section{Conclusion and perspectives}

In this paper, we have introduced an Ellipsoidal Statistical Model for gas mixtures following the moments relaxation method introduced in $[7,8,10]$. The resulting model fulfills two important features. Firstly, it enjoys the fundamental properties of the Boltzmann operator ( $\mathrm{H}$ theorem, nonnegativity of the distribution function, conservation laws, indifferentiability condition, ...) which is only satisfied by a few models [1]. Moreover, its construction is based on the right hydrodynamic coefficients and the model is able to reach a certain range of Prandtl numbers. This range includes the correct Prandtl number if one has the indifferentiability condition.

For concrete applications, this methodology should be generalized to the situation of polyatomic gases in order to fit the Prandtl number. Hence the following step of this work should be the derivation of an Ellipsoidal Statistical Model for polyatomic gas mixtures. For example the atmosphere can be considered as a binary mixture of Oxygen and Nitrogen. In that case, the distribution function depends on an additional continuous variable, the internal energy [5], collecting rotational, vibrational, translational energy and belonging to $\mathbb{R}_{+}$. In [8], we have derived from the methodology described in this paper an ESBGK model for a polyatomic single gas. So by taking advantage of this work, we plan to generalize this construction to a gas mixture framework. Before this, the Chapman-Enskog expansion for polyatomic gas mixtures has to be clarified. This work is postponed to a future paper.

\section{REFERENCES}

[1] P. Andries, K. Aoki, and B. Perthame, A consistent BGK-type model for gas mixtures, J. Stat. Phys., 106(5/6), 993-1018, 2002.

[2] P. Andries, P. LeTallec, J.P. Perlat, and B. Perthame, Entropy condition for the ES BGK model of Boltzmann equation for mono and polyatomic gases, Eur. J. Mech. (B fluids), 19, 813-830, 2000.

[3] P.L. Bhatnagar, E.P. Gross, and M. Krook, A model for collision processes in gases, Phy. Rev., 94, 511-524, 1954.

[4] M. Bisi and G. Spiga, On a kinetic BGK model for slow chemical reactions, Kin. Rel. Model., 4(1), 2011.

[5] J.F. Bourgat, L. Desvillettes, P. Le Tallec, and B. Perthame, Microreversible collisions for polyatomic gases and Boltzmann's theorem, Eur. J. Mech. (B-Fluids), 13(2), 237-254, 1994.

[6] S. Brull, Un modèle ES-BGK pour des mélanges de gaz, Comptes Rendus Math., 351, 775-779, 2013. 
[7] S. Brull and J. Schneider, A new approach of the ellipsoidal statistical model, Cont. Mech. Thermodyn., 20(2), 63-74, 2008.

[8] S. Brull and J. Schneider, On the ellipsoidal statistical model for polyatomic gases, Cont. Mech. Thermodyn., 20(8), 489-508, 2009.

[9] S. Brull and J. Schneider, Derivation of a BGK model for reacting gas mixtures, Commun. Math. Sci., 12(7), 1199-1223, 2014.

[10] S. Brull, V. Pavan, and J. Schneider, Derivation of BGK models for mixtures, Eur. J. Mech. (B-Fluids), 33, 74-86, 2012.

[11] S. Chapman and T.G. Cowling, The Mathematical Theory of Non Uniform Gases, Third Edition, Cambridge Univ. Press, Cambridge, 1970.

[12] S.R. de Groot and P. Mazur, Nonequilibrium Thermodynamics, North-Holland, Amsterdam, 1962.

[13] V. Garzo, A. Santos, and J.J. Brey, A kinetic model for a multicomponent gas, Phys. Fluids, A 1 N 2, 380-383, 1989.

[14] M. Groppi, S. Rjasanow, and G. Spiga, A kinetic relaxation approach to fast reactive mixtures: Shock wave structure, J. Stat. Mech. Theory. Exp., P10010, 2009.

[15] M. Groppi and G. Spiga, Kinetic approach to chemical reactions and inelastic transitions in a rarefied gas, J. Math. Chem., 26, 197-219, 1999.

[16] M. Groppi and G. Spiga, A Bhatnagar-Gross-Krook approach for chemically reacting mixtures, Phys. Fluids, 16(12), 4273-4284, 2004.

[17] P.L. Gross and M. Krook, Model for collision processes in gases: Small-amplitude oscillations of charged two-component systems, Phys. Rev., 102(3), 593-604, 1956.

[18] B.B. Hamel, Two-fluid hydrodynamic equations for a neutral, disparate-mass, binary mixture, Phys. Fluids, 9(12), 11-22, 1966.

[19] L.H. Holway, New statistical models for kinetic theory: Methods of construction, Phys. Fluids, 9, 1958-1673, 1966.

[20] M. Junk, Maximum entropy for reduced moment problems, Math. Mod. Meth. Appl. Sci., 10(7), 1001-1025, 2000.

[21] S. Kosuge, Model Boltzmann equation for gas mixtures: Construction and numerical comparison, Eur. J. Mech B/Fluids, 28(1), 170-184, 2009.

[22] D. Levermore, Moment closure hierarchies for kinetic theories, J. Stat. Phys., 83(5-6), 10211065, 1996.

[23] J. Schneider, Entropic approximation in kinetic theory, M2AN, 38(3), 541-561, 2004. 